\title{
CONTRA LAS INTERPRETACIONES SIMPLISTAS DE LOS RESULTADOS DE LOS EXPERIMENTOS REALIZADOS EN EL AULA
}

\author{
LUCAS, A M., GARCÍA-RODEJA GAYOSO, I. \\ Science Education Unit, Centre for Educational Studies, King's College London, University of London. \\ Departamento de Didáctica das Ciencias Experimentais, Escola Universitaria do Profesorado de EXB \\ Universidade de Santiago de Compostela.
}

\section{SUMMARY}

This paper is about the importance of the students' interpretation of experiments made in the school. Examples are given of the students' interpretations of specific activities, and possibles classroom strategies bases on these interpretations are suggested.

\section{INTRODUCCIÓN}

En el presente artículo se resume la experiencia llevada a cabo en la Escuela Universitaria del Profesorado de EGB de Santiago de Compostela, referente a un taller dirigido por el profesor A. Lucas, al que han asistido profesores del Departamento de Didáctica de las Ciencias Experimentales.

En el taller, se realizan una serie de actividades, sencillas y de utilización frecuente en el aula, ya que muchas se proponen tanto en libros de texto de EGB del Ciclo Superior, como en proyectos curriculares de diversos países, para introducir conceptos fundamentales en la Enseñanza de las Ciencias como difusión de gases, transporte del agua en las plantas, formación de precipitados, etc.

Actualmente en muchos países los cursos de ciencias incluyen objetivos sobre el entendimiento de la evidencia científica. Por ejemplo, en Inglaterra desde 1988 hay un programa para la renovación completa de los cursos para alumnos de 5 a 16 años de edad. Este pro* grama será obligatorio, y en las ciencias incluye un grupo de objetivos sobre "exploraciones de las ciencias". (Great Britain, Department of Education and
Science 1989). Los alumnos tendrán que considerar las interpretaciones de evidencias, observaciones, inferencias, diseños de experiencias, etc.

Este tipo de objetivos también se tienen en cuenta en otros países. Ver, por ejemplo, el documento consultivo del "Curriculum Development Centre" Australiano (1988):

Science is a way of viewing the world. It is based on particular methods of enquiry, ways of thinking, attitudes and traditions.

(La Ciencia es una forma de ver el mundo. Se basa en métodos de investigación específicos, formas de pensamiento, actitudes y tradiciones).

El documento australiano recalca los aspectos de pensamiento que utilizan los científicos. En EEUU los objetivos de los "procesos de las ciencias" son semejantes y se han escrito muchos artículos de investigaciones sobre la comprensión de los alumnos. Ver, por ejemplo, Sneider y otros (1984) y Lucas y Tobin (1987), artículo en el que se hace una crítica de los su- 
puestos que utilizan los primeros autores. Otros autores han tratado las dificuldades y la importancia de interpretar las actividades experimenatales en el aula. Por ejemplo, Hodson (1985) ha examinado los trabajos en este campo; Wood-Robinson (1984) ha comentado Los problemas en biología; Harris y Taylor (1986) ha señalado las dificuldades en el contexto de 'Discovery Learning' (enseñanza por descubrimiento). Proximamente se considerarán diversos aspectos de la relación entre la filosofía de las ciencias y la ciencia en el aula en las ediciones especiales de las revistas Educational Philosophy and Theory, Interchange, Studies in Philosophy and Education, y Synthese en 1988 y 1989 . Ver también Solomon (1988), Elkana (1970), Licas (1977), Smolicz y Nunan (1975), Millar (1987). En este trabajo, sin embargo, consideramos ejemplos concretos, y no discutimos los principios filosófícos.

En España, también tenemos ejemplos en donde se formulan este tipo de objetivos. En el Proyecto para la Reforma, uno de los objetivos que se señalan dentro de la formación común del Bachillerato es el desarrollo del pensamiento científico. Entre los objetivos de Ia formación específica del Bachillerato de Ciencias de la Naturaleza se proponen:

- Familiarizar al estudiante con los principios de la Ciencia.

- Adiestrar en los métodos de investigación científica.

- Fomentar la actitud reflexiva frente a los problemas científicos, evitando la precipitación y el operativismo.

Todos estos objetivos se reflejan en muchas de las actividades ya incluidas en cursos de ciencias. Sin embargo, a nuestro entender, muchos profesores y maestros pueden tener dificultades porque algunas đe estas actividades dan datos que se explican de más formas que la típica interpretación dada por profesores y libros de textos.

Por ejemplo, la clásica demostración de la fotosíntesis: Se cubre la mitad de una hoja de una planta viva con plástico negro o papel de aluminio, se expone a la luz y después de una hora se hace la prueba del almidón. El almidón se encuentra sólo en la mitad de la hoja que no se ha cubierto con el papel de aluminio o el plástico. La interpretación común, es que la luz es necesaria para que se realice la fotosíntesis. Hay, sin embargo, otras interpretaciones: el plástico negro produce más calor, o al cubrir los estomas no hay intercambio de gases, o el plástico contiene una sustancia tóxica, etc. La interpretación correcta del efecto de la luz no es la única. En general, se enseña a los alumnos a que consideren sólo una explicación y a que la acepten.

El mayor problema, en este tipo de actividades es que el profesor al conocer ya la respuesta correcta no considera otras posibles interpretaciones. La actividad, en este caso, no es una investigación, sino únicamente una demostración del efecto de la luz.

Pensamos que es esencial que, tanto los alumnos como los profesores, practiquen esta forma de pensamiento crítico al interpretar los datos experimentales de las investigaciones.

\section{1. ¿CÓMO ES EL MOVIMIENTO DEL AGUA EN LAS PLANTAS?}

Material: Plantas de apio. Recipiente. Agua. Eosina.

Una experiencia que se cita con frecuencia para demostrar el mecanismo de ascenso del agua en las plantas es la que se realiza con plantas de apio introducidas en re. cipientes con agua y algún colorante como la eosina. Con esta actividad se pretende que el alumno aprecie la existencia de unas estructuras especializadas en conducir el agua.

Sin embargo, por la simple observación de las hojas coloreadas, o por la tinción de determinadas partes de una sección del tallo, el alumno puede hacer distintas interpretaciones .

\section{Por ejemplo:}

"El agua sube por todas las partes de la planta, y sólo se tiñen determinadas estructuras por una reacción específica entre la eosina y alguna sustancia presente en dichas estructuras". Recordemos que otra práctica habitual en las escuelas es la de reconocer la presencia de almidón en las plantas al añadir yodo.

Cuando enseñamos este tema, deberíamos ser conscientes de estas posibles interpretaciones del alumno, buscando pruebas que eliminen las interpretaciones erróneas y apoyen la interpretación válida, teniendo en cuenta la diferencia que hay entre la posición deI alumno, cuyas explicaciones se basan casi exclusivamente en la observación y el profesor que lo hace mediatizado por sus conocimientos previos. Es importante que los alumnos piensen sobre la validez de las interpretaciones de los fenómenos, y que los profesores no acepten sin discusión crítica, la interpretación válida.

En esta experiencia, una posible estrategia sería observar cómo la parte inferior del tallo en contacto directo con el colorante se tiñe por completo, mientras que si realizamos un corte en la parte superior del mismo, sólo estarán coloreadas determinadas zonas. De esta forma se elimina la posibilidad de que Ia eosina sea una tinta selectiva.

\section{2. ¿CUÁL ES LA FUNCIÓN DEL AGUA EN LAS REACCIONES QUIMICAS?}

Material: Tubos de ensayo. Cristales de $\mathrm{Pb}\left(\mathrm{CH}_{3} \mathrm{COO}\right)_{2}$ $\mathrm{Pb}\left(\mathrm{NO}_{3}\right)_{2}$ y de $\mathrm{KI}$. Agua.

Normalmente, en la escuela, este tipo de reacciones se realizan con sustancias que ya están en disolución, con lo que se priva al alumno de que piense en el papel que tiene el agua .

Con la siguiente experiencia, se pretende inducir al 
alumno a reflexionar sobre el papel del agua en la reacción.

La experiencia consiste en agitar cristales de acetato de plomo y de yoduro potásico, y una vez agitados observar que no se ha producido ningún cambio de color. Posteriormente al añadir unas gotas de agua, se volverá amarillo.

$$
\mathrm{Pb}\left(\mathrm{CH}_{3} \mathrm{COO}\right)_{2}+2 \mathrm{KI} \rightarrow Æ \mathrm{PbI}_{2}+2 \mathrm{~K}\left(\mathrm{CH}_{3} \mathrm{COO}\right)
$$

Estas observaciones, pueden llevar al alumno a considerar el agua como un reactivo, o como un catalizador de la reacción. Es interesante en este momento que los alumnos expresen sus propias explicaciones.

En la segunda parte de la actividad se agita nitrato de plomo y yoduro potásico y se comparan los resultados con los de la experiencia anterior.

$$
\mathrm{Pb}\left(\mathrm{NO}_{3}\right)_{2}+2 \mathrm{KI} \rightarrow \notin \mathrm{PbI}_{2}+2 \mathrm{KNO}_{3}
$$

Esta vez, el precipitado se produce antes de añadir el agua. El que la reacción se produzca sin agua llevará a algún alumno a sugerir otra experiencia secando previamente los cristales (recordemos que el nitrato de plomo es higrosc6pico). De esta forma los alumnos se ven obligados a pensar en la funcion del agua.

\section{DIFUSIÓN DE GASES}

Material: Tubo de cristal de Im Tapones. Algodón. Chinchetas. Disolución concentrada de HCl. Disolución concentrada de $\mathrm{NH}_{3}$.

Esta actividad se propone por ejemplo, en el proyecto CLIS o en el Nuevo Manual de la Unesco para la Enseñanza de las Ciencias, con el objeto de mostrar la difusión de los gases.

Para la realizacion de la experiencia, se coloca en un tapón, algodón con unas gotas de cloruro de hidrógeno y en otro, algodón con amoníaco y se tapan con ellos los extremos del tubo ( fig. 1). En unos minutos se observa la formación de una nube blanca, más próxima al extremo donde hemos colocado el tapón con el algodón con cloruro de hidrógeno.

$$
\mathrm{HCl}_{(\mathrm{g})}+\mathrm{NH}_{3(\mathrm{~g})} \rightarrow \& \mathrm{NH}_{4} \mathrm{Cl}_{(\mathrm{s})}
$$

La discusión se centra, en por qué, la nube se forma más cerca del tapón con cloruro de hidrógeno, intuitivamente la tendencia es a pensar que la nube se debería formar en el centro y que esta posición puede deberse únicamente a las condiciontes en las que se realiza la experiencia.

El profesor conoce a priori la explicación a este fenómeno (la velocidad de difusión del amoníaco es mayor que la del cloruro de hidrógeno) pero el alumno, sin ese conocimiento previo, puede encontrar otras posibles explicaciones, por ejemplo:

"La nube se forma en el extremo porque la concentración del amoníaco es mayor".
Si posteriormente se pone hielo en el extremo donde se colocó el algodón con el amoníaco, la nube cambia de posición moviéndose hacia el extremo con hielo. EI cambio de temperatura puede ayudar para que la interpretación se haga en términos de difusión desechando otras alternativas.

figura 1

Difusión de gases

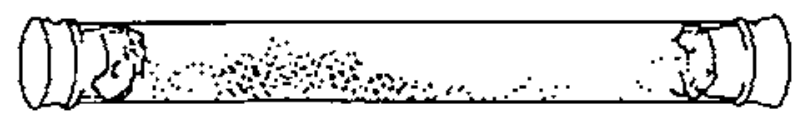

$\mathrm{HCl}$

$\mathrm{NH}_{3}$

\section{REFLEJO PUPILAR A LA LUZ}

\section{Material: Un espejo y una linterna.}

Se trata de tapar un ojo completamente y enfocar la linterna en el otro. Cuando se observe algún cambio se destapa el ojo y se comparan las dos pupilas.

El tamaño de las dos pupilas no es tan diferente como podría haber esperado el alumno.

Debemos hacerle reflexionar ante este hecho ayudándole a separar el fenómeno de que la pupila se contrae con la luz y la respuesta que se produce a través del núcleo nervioso que mandará el impulso nervioso hacia los dos ojos, excitando los dos esfínteres pupilares y disminuyendo la abertura de las dos pupilas.

Es interesante realizar este tipo de actividades con niños de diez u once años, edad en la que ya se comienza a hacer predicciones. Es también importante discutir las pruebas que se van a realizar con el alumno, ya que el alumno debe entender que los experimentos se realizan por alguna razón y éste es un paso que se olvida con demasiada frecuencia.

\section{INTERPRETACIÓN DE SECCIONES}

\section{Material: Manzanas y naranjas.}

En materias como la Biología no se suele tener en cuenta las dificultades que puede suponer para los alumnos la interpretación de estructuras bidimensionales que representan estructuras tridimensionales. Así, en los libros de texto, nos encontramos secciones de órganos para el estudio de su estructura, de los que el alumno tiene que interpretar la estructura tridimensional; Io mismo ocurre en el estudio de la disposición de las células de determinados tejidos, a partir de la observación de preparaciones al microscopio. 
Sin embargo, para la interpretación de estas estructuras es necesario un aprendizaje previo.

La actividad consiste en practicar cortes en distintas direcciones en objetos tan familiares como naranjas o manzanas. Se le indica al alumno la dirección de los cortes que se van a realizar y se le dice que dibuje las secciones que espera cncontrar después de realizar el corte. Posteriormente comparamos sus predicciones con la sección que resulta (fig. 2 , fig. 3 ).

Esta actividad nos pone de manifiesto, la dificultad qué entraña la interpretación de secciones, ya que generalmente los dibujos de los alumnos tienen poco que ver con el resultado real del corte.

En la siguiente parte de la actividad, se muestran figuras geométricas bidimensionales como una esfera o una elipse a partir de las cuales el alumno debe predecir a qué figura de tres dimensiones podrían corresponder cada sección. Así, tanto la elipse como el círculo podrían corresponder a una esfera, un cono, un cilindro, etc.

Una estructura bidimensional puede representar diferentes estructuras tridimensionales, de ahí que se deba ayudar al estudiante a interpretar las estructuras con tres dimensiones a partir de estructuras bidimensionales.

figura 2

1 y 2 Errores frecuentes. 3 Sección real.
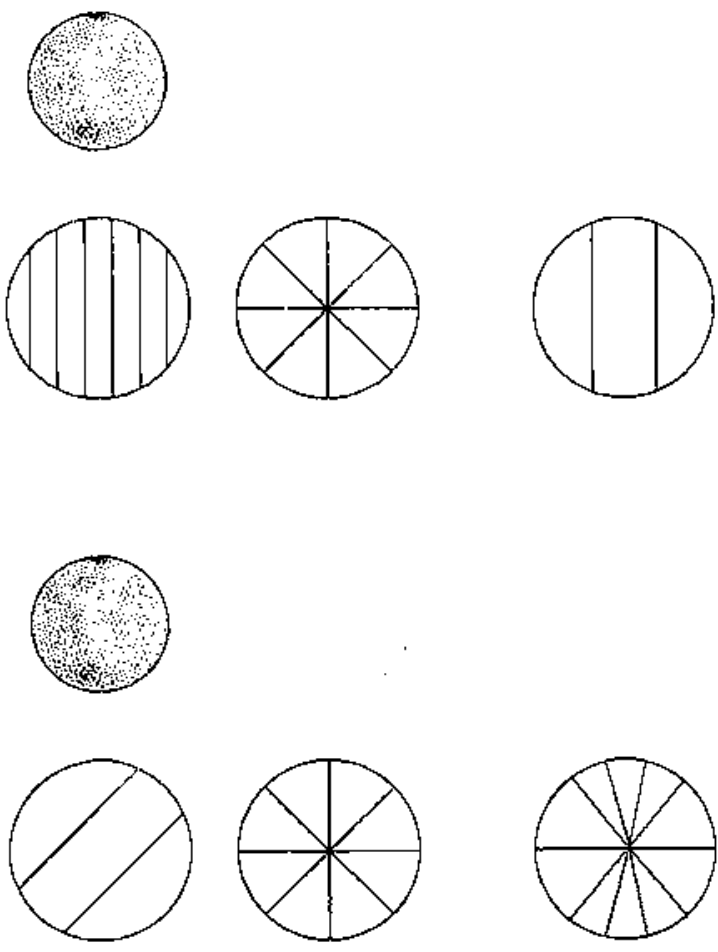

2

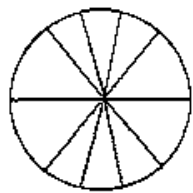

3

\section{UN TRUCO QUE NOS HACE PENSAR}

Material: Un huevo duro sin cascara. Una botella con el cuello de unos $4 \mathrm{~cm}$. Papel. Cerillas.

El huevo se coloca en la parte superior de la botella. Al introducir un papel ardiendo dentro de la misma, el huevo cae dentro. Una explicación frecuente, que dan los alumnos es la siguiente:

"El huevo entra en la botella porque en la combustión del papel se consume el oxígeno produciéndose entonces una descompresión ".

En esta interpretación no se tiene en cuenta la formación de $\mathrm{CO}_{2}$ y $\mathrm{H}_{2} \mathrm{O}$ en la combustión.

Si estamos atentos un instante antes de que el huevo caiga, observaremos que rebota un poco antes de caer. Si sacamos el huevo de la botella soplando estaremos dando una pista que ayudará a llegar a la interpretación válida ( el aire se expande y sale al calentarse; una vez que se apaga el papel, el aire que queda en la botella se enfría y se comprime provocando la succión del hucvo, por las diferencias de presiones).
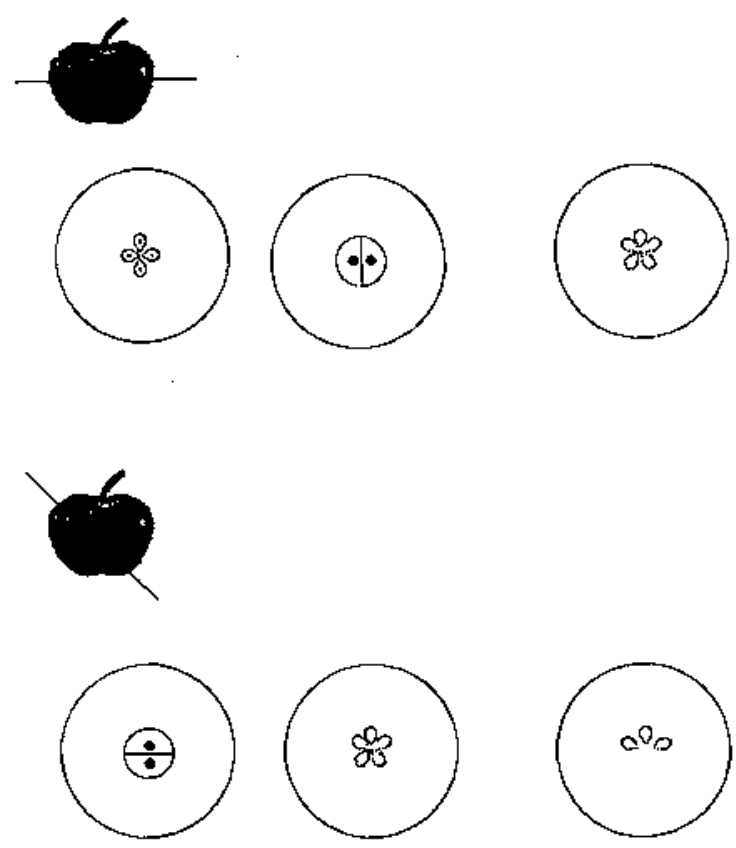

2

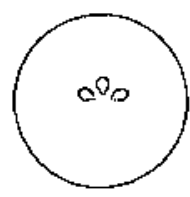

3 
Estas interpretaciones alternativas no están sólo en la mente del alumno sino que también se ponen de manifiesto en los libros de texto. La clásica experiencia de la vela encendida que flota sobre un recipiente y que al cubrirla con un vaso se apaga y provoca el ascenso del agua en el vaso, se utiliza frecuentemente en la enseñanza para demostrar la composición del aire, incluso en un aspecto cuantitativo, refiriéndose a que el ascenso del agua que tiene lugar una vez que se apaga se corresponde con la proporción de oxígeno en el aire:

"Si en el aire hay un quinto de oxígeno, el agua subirá un quinto del volumen del recipiente una vez que se haya apagado la vela".

Una prueba sencilla, para demostrar que no se consume todo el oxígeno, sería introducir un ratón y realizar la experiencia. El ratón sigue respirando, aún después de que el fuego de la vela se haya extinguido; no hay duda entonces de que queda oxígeno.

\section{COMENTARIO FINAL}

Somos de la opinión de que la enseñanza de las ciencias en los colegios debe incluir tanto aspectos metodológicos como conceptuales. Pensamos que la forma de enseñar del profesor, cuando el profesor no hace co. mentarios sobre el tipo de razonamientos que se utilizan, puede Ilevarnos a que Ios alumnos aprendan formas incorrectas de interpretación de evidencias científicas.

Por ejemplo, hay una demostración en química que muestra cómo los óxidos de los metales forman soluciones básicas y los $6 x$ idos de los no metales forman soluciones ácidas. El profesor quema un poco de sodio en un recipiente de oxígeno, y obtiene el color típico de las sustancias básicas con el tornasol. De la misma forma utiliza azufre y obtiene con el tornasol, color rojo. La experiencia se realiza poniendo un solo ejemplo de metales y de no metales, y se dice que esta experiencia confirma el principio. Sin embargo, utili-

\section{REFERENCIAS BIBLIOGRÁFICAS}

CURRICULUM DEVELOPMENT CENTRE, 1988. Science for everybody: towards a national science statement. (Curriculum Development Centre, Canberra).

CHILDREN'S LEARNING IN SCIENCE PROIECT, 1987. Approaches to Teaching the Particulate Theory of Matter. (University of Leeds).

GREAT BRIT AIN, DEPARTMENT OF EDUCATION AND SCIENCE, 1989. Science in the National Curriculum. (Her Majesty's Stationery Office, London). zando Ia misma lógica, se puede probar una regla falsa: "los metales arden con una llama amarilla y los no metales arden con una llama violeta". Los profesores al no considerar esta posible interpretación, permiten que algunos alumnos aprendan de la experiencia, una regla sobre el color de las llamas de los elementos que no es válida.

Para que los alumnos puedan aprender que la ciencia progresa a través de las interpretaciones que se hacen de los hechos y que esto conlleva la eliminación de otras posibles interpretaciones, es necesario que los alumnos piensen en más de una interpretación y que busquen la forma de eliminar algunas. Así aprenderán que los hechos científicos no hablan por sí solos.

Es de gran importancia ser conscientes de que las experiencias en el aula no son experiencias reales: el profesor conoce a priori la explicación, e interpreta la demostración desde este conocimiento. Cuando los alumnos interpretan los datos, si los profesores no discuten otras interpretaciones, los alumnos confundirán la naturaleza de la evidencia científica.

Sugerimos:

- Pensar en las interpretaciones alternativas.

- Buscar las evidencias para cada posible interpretación.

- Distinguir las evidencias de las inferencias.

- Distinguir las verdaderas investigaciones (los profesores y los alumnos desconocen de antemano los resultados) de las demostraciones (los profesores conocen los principios científicos, y utilizan las demostraciones para ejemplos concretos).

- Ayudar a los alumnos a pensar sobre la evidencia de las interpretaciones.

Finalmente y a un nivel más complejo, recordar nuestras opiniones de cómo la filosofía y la estructura de la ciencia influye en cómo y qué se enseña. (Lucas 1986, p. 191).
ELKANA, Y., 1970. Science, philosophy of science and science teaching, Educational Philosophy and Theory, 2, pp. 15. 35.

HARRIS, D, y TAYLOR, M., 1986. Discovery learning in school science: the myth and the reality, en P.G. Taylor (ed.), Recent Developments in Curriculum Studies. NFERNelson, pp. 169-187.

HODSON, D., 1985. Philosophy of science, science, and science education, Studies in Science Education, 12, pp. 25-57. 
LUCAS, A. M., 1977. Should 'science' be studied in science courses?, Australian Science Teachers Journal, 23(2), pp. $31 \cdot 37$.

LUCAS, A. M., 1986. Tendencias en la investigación sobre la enseñanza/aprendizaje de la biología, Enseñanza de las Ciencias, 4, pp. 189-198.

LUCAS, A. M., y TOBIN, K., 1987. Problems with 'control of variables' as a process skill, Science Education, 71, pp. 685-690.

MILLAR, R., 1987. Towards a role for experiment in the science teaching laboratory, Studies in Science Education, 14, pp. 109-118.
SMOLICZ, J. J. y NUNAN, E. E. 1975. The philosophical and socialogical foundations of science education: the demythologizing of school science, Studies in Science Education, 2, pp. 101-143.

SNEIDER, C., KURLICH, K., PULOS, S. y FREIDMAN, A. 1984. Learning to control varjables with model rockets: a neo-Piagetian study of learning in field settings, Science Education, 68, pp. 463-484.

SOLOMON, J., 1988. Learning through experiment, Studies in Science Education, 15, pp. 103-108.

WOOD-ROBINSON, C., 1984. How many experimental conclusions are a 'con'?, Journal of Biological Education, 18, pp. 1-2. 